\title{
Integration Factor Construction to Improving Aesthetic, Physical and Mechanical Properties of Double Layer Woven Fabrics by Denting and Stitches Systems
}

\author{
El Sayed A Elnashar ${ }^{1 *}$ and GV Bashkova ${ }^{2}$ \\ ${ }^{1}$ Kafrelsheikh University, Egypt \\ ${ }^{2}$ Ivanovo State Polytechnic University, Russia
}

*Corresponding author: El Sayed A Elnashar, Textiles \& Apparel, Kafrelsheikh University, Egypt.

\begin{abstract}
Clothing for garments, upholstery and geotextiles are designed through integration factor construction (IFC) are differentiated the system of reed denting; the decrease in the (IFC) means that the open porosity for double-layer fabrics increases and at the same time the air permeability also increases. The properties of fabrics were analyzed by determining the efficiency of fabric integration factor construction (IFC). The woven fabric multi-layer structure, the warp and weft densities, and the type of weave are factors of a woven fabric, which as porous material enables to transmit air, heat energy, and liquid perspiration. Several methods considering thread distributions have been developed to determine the woven fabric's aesthetic, physical and mechanical properties. A mathematical model based on an ideal geometry of the porous structure of a multi-layer woven fabric has been developed.
\end{abstract}

Keywords: Multi-layer structure; Mechanical and physical; Aesthetic properties

\section{Introduction}

The potential of denting reed systems in textiles design, through denting is one of the most widely used of fabrics design in the world. Reed is the active ingredient is a global commodity with annual machinery of textile production. The denting and stitches systems are critical in determining the quality multi-layer for garments clothing, upholstery and geotextiles are designed throw integration factor construction (IFC) are differentiated the system of reed denting; the decrease in the (IFC) means that the open porosity for double-layer fabrics increases and at the same time the air permeability also increases [1-3]. Bending stiffness is a property of fabrics that can influence fabric drape and therefore the aesthetic characteristics of garments and clothing comfort as well [4].

The numerous investigations proved that the properties of bending, creasing and draping ability are all related to the factors of fabric structure [5]. Tested and analyzed some cotton, wool, silk and man-made fabrics and they concluded that the bending, creasing and draping are correlated to fabric type. Dr. Jing [6] has analyzed the structures of lightweight worsted fabrics. It has been investigated some characteristics of worsted fabric such as wrinkle resistance, blend resistance, drapeability and air permeability. Using the gray interrelation theory to deal with the data of structure parameters and wearing characteristics target and analyzed the relationship between the wearing fabric characteristics and structure parameters. Also, drapeability was evaluated on the basis of drape coefficient, number of folds and maximum and minimum amplitude [7]. The results have shown that there is a great influence of material type and yarn structure on fabric rigidity.

\section{Methods of stitching}

Multi-layer woven fabric consists of at least two layers which are woven one above the other and stitched together. Double woven fabric contains two systems of warp, face and back, and two systems of weft. The fabric can be called either two-ply fabric or double 
fabric [8]. In this way, the aim if this paper is to further investigate the influence of integration factor construction to improving aesthetic, physical and mechanical properties of double layer woven fabrics by denting and stitches systems. Textile structure is in this case usually represented by its porosity. A number of theoretical and experimental methods exist for the determination of porosity. Every method includes some simplifying assumptions which cause inaccuracy. Generally, the porosity indicates how much air contains a textile material with a given warp-ends density distribution. Further details about the array of pores in textiles, such as the pore size, shape, and arrangement are very important for the description of physical properties of multi-layer woven fabrics $[2,3,9,10]$ and the air flow through textile materials. The subjects of this research are:

a. To study the integration factor construction models of multi-layer woven fabrics considering the flow interaction between denting and thread distribution; this research modifies an existing three-dimensional model to include in it the simulation capabilities, and to verify this model with the fabrication of structural composites.

b. To characterize the eight-harness fabric (for use in processing) by the stitches systems, the thread distribution in the multi-layer woven fabric, physical and mechanical properties.

c. To use the model for analysis of the manufacturing process where this model should enable the user to design the best process for manufacturing aesthetic of high quality.

\section{Materials and Methods}

Experiments were carried out on woven fabrics, which were constructed according to the setting theory. The following yarns were used: combed warp yarns of 20.4 Tex for the upper fabric, 39.4 Tex for the lower fabric, and weft yarns of 39.4 Tex for the upper and lower fabrics, spun from $100 \%$ cotton staple fiber (Giza 70) on a ring spinning machine. The packing factor and the factor of thread flexibility were determined according to literature. The parameters of the woven fabric structure accepted by us are the weave factor and the fabric density. All variables used for determining the weave factor were collected (maximum densities, setting thread densities, actual thread densities and relative densities). All fabric samples were woven with shedding mechanism "Stauble" on weaving machine under equal technological conditions [3]. The two-layer woven fabrics (100\% cotton) were designed and manufactured with variations in distribution of warp types as given in Table 1.

Table 1: Description of the experimental weaves.

\begin{tabular}{|c|c|c|c|c|c|c|}
\hline $\begin{array}{c}\text { Woven } \\
\text { Fabrics }\end{array}$ & $\begin{array}{c}\text { Weave Pattern of } \\
\text { Upper Fabric }\end{array}$ & $\begin{array}{c}\text { Weave Pattern of } \\
\text { Lower Fabric }\end{array}$ & $\begin{array}{c}\text { Linear Density of } \\
\text { Upper Warp,Tex }\end{array}$ & $\begin{array}{c}\text { Linear Density of Upper } \\
\text { and Lower Wefts, Tex }\end{array}$ & $\begin{array}{c}\text { Linear Density of } \\
\text { Lower Warp, Tex }\end{array}$ & $\begin{array}{c}\text { Fabric Density, } \\
\text { Warp×Weft/Per Cm }\end{array}$ \\
\hline I & Plain1/1 & Plain1/1 & 20.4 & 39.4 & 39.4 & $48 \times 18$ \\
\hline II & Twill 3/3 & Plain1/1 & 20.4 & 39.4 & 39.4 & $48 \times 18$ \\
\hline II & Satin 6 weft & Plain1/1 & 20.4 & 39.4 & 39.4 & $48 \times 18$ \\
\hline
\end{tabular}

\section{Results and Discussion}

In textiles mathematics, an integrating factor concretion (IFC) is a function that is chosen to facilitate the solving of a given equation involving differentials. It's commonly used to solve ordinary differential equations but is also used within multivariable as elements construction in double-layer fabrics calculus when multiplying through by an integrating factor allows an inexact differential to be made into an exact differential for aesthetic, physical and mechanical properties, which can then be integrated to give a scalar field. This is especially useful in prediction of textiles properties such as thermodynamics where temperature becomes the integrating factor that makes entropy an exact differential as the following Figures (1-8).

\section{Neseas}

Figure 1: Denting regular system as single (1/1 plain weave for lower as $2 /$ gate in reed for upper layer fabric).

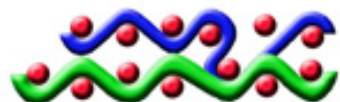

Figure 2: Denting regular system as double (cross-section plain weave $1 / 1$ for two layers).

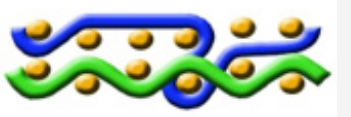

Figure 3: Fabric cross-section as double (1/1 plain weave for lower and twill weave $3 / 3$ for upper and plain weave $1 / 1$ for lower fabric).

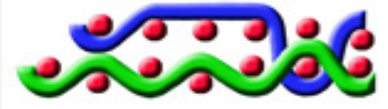

Figure 4: Fabric cross-section (1/1 plain weave for lower and satin weave 6 weft for upper fabric and plain weave1/1 for lower fabric).

\section{revesese}

Figure 5: Red dents system as single (1/1 plain weave for lower as $3: 1 / 2$ gate in reed for upper).

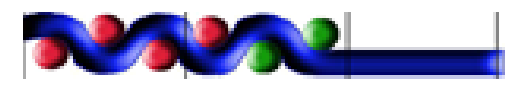

Figure 6: Red denting system as single (1/1 plain weave for lower as 3:3:0/3gate in reed for upper). 


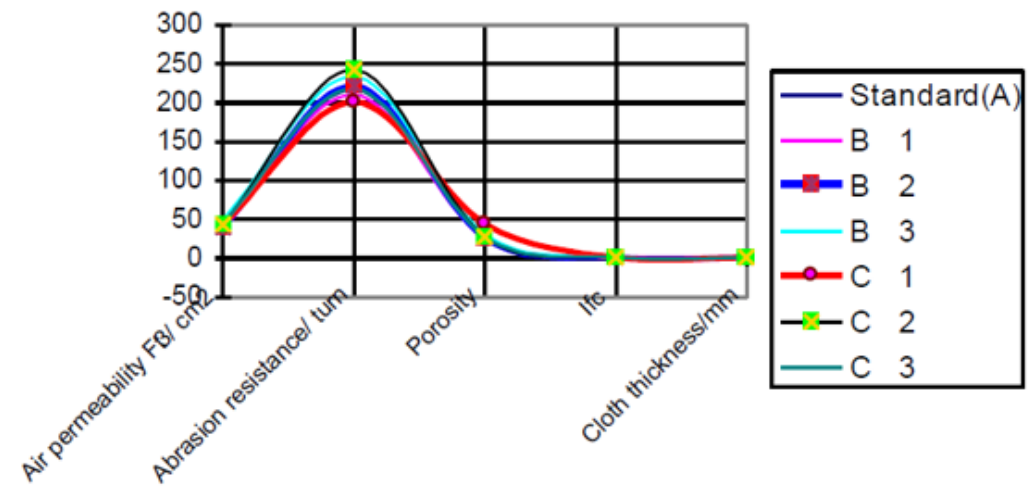

Figure 7: Graph of double-layers fabric's properties with plain 1/1 in upper cloth.

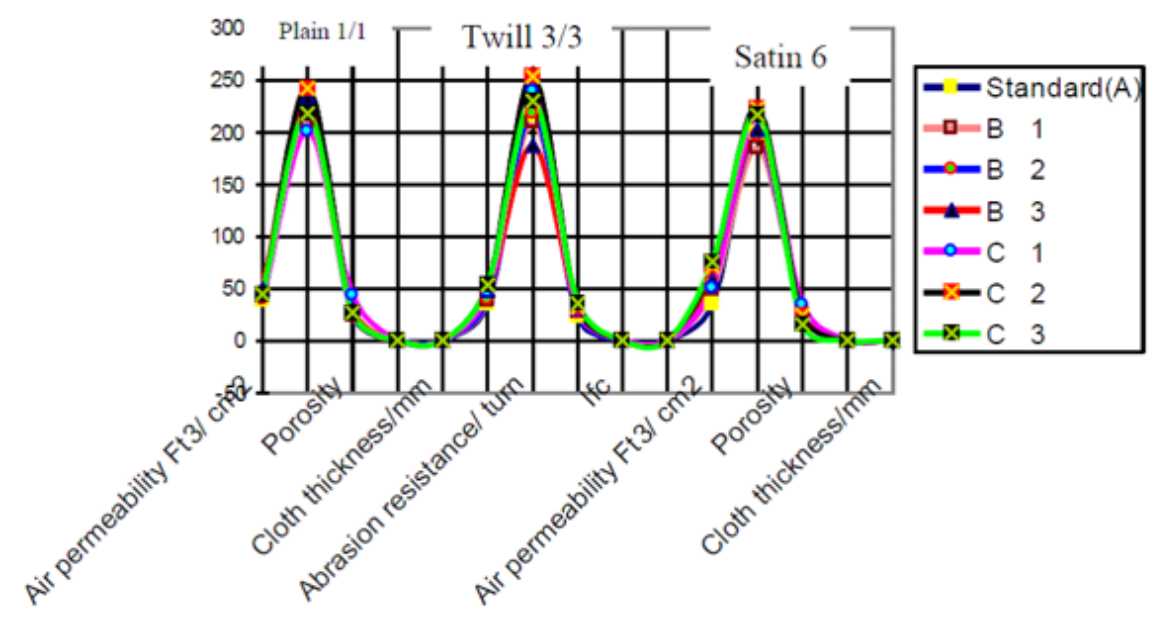

Figure 8: Graph comparison of double-layers fabric's properties with plain 1/1, twill $3 / 3$ and satin 6 wefts in upper cloth

Stitch weave is satin 6 wefts between the layers. Structure of textile changes by influence of denting system is possible classified as a horizontal increase of the porosity, removing of free yarn section. Yarn is interlaced very closely in a plain weave, in twill or satin weaves relative removing of yarns cause an increase of its porosity predominantly in a vertical direction. Flowing air cause a move of not interlaced parts of yarns-floats. In textiles depends on the length of floats. So, the horizontal increase of porosity can result in a considerable increase of aesthetic, physical and mechanical properties of double layer woven fabrics. The experimental study was carried out as a new approach to predict the parameters of integration factor construction by using a factor analysis based on the mathematical model developed by us. Physical and mechanical properties are an important material property which knowledge is required in various flow simulations while processing composites. This quantity is defined as the property of a stitch's systems material, which characterizes the ease with which a fluid is forced to flow through the material under an applied pressure gradient. Both, the steady state and the impact mechanical properties measurement techniques were used in this work. The model higher standard was used as a process analysis tool. This enabled the user to determine such important process parameters as the location and type of injection ports, as well as the, physical and mechanical properties and location of the high- aesthetic textile products. A process for a three-stiffener composite panel was proposed by using a denting system as shown in Table 2 (B, C, D). This array evolved from the variation of the process parameters while modeling several different composite panels [3].

$$
I F C=\sum(\alpha X W) / n,(1)
$$

where: $\alpha$ - balance factor of woven construction; $\mathrm{W}$ - width of stripe (density); $\mathrm{n}$ - number of width stripes (density).

Then, $K=C n \sqrt{N},(2)$

for a direct system, $\mathrm{C}$ - is the constant for material, $(0.04126$ for Tex); $\mathrm{n}$ - number of threads per inch; $\mathrm{N}$ - yarn count.

Calculate the integration factor construction in regular Red dents system with plain weave $1 / 1$ for double-layer fabrics as $5: 4$ yarns:

First in 5 yarns (A).

$K_{\text {up } 1}=\operatorname{Cn} \sqrt{N}=0.04126(3 \times 10 \times 2.54) \sqrt{20.4}=14.2 ; K_{\text {up } 2}=0.04126 \times 18 \times 2.54 \times \sqrt{39.4}=11.84$

$$
\alpha_{u p=} K_{u p 1 /} K_{u p 2}=14.2 / 11.84=1.1993
$$


$K L_{\text {w1 }}=0.04126(2 \times 10 \times 2.54) \sqrt{39.4}=13.156 ; K L_{w 2}=0.04126(18 \times 2.54) \sqrt{39.4}=0.04126 \times 45.72 \times 6.2769419=11.840$

$$
\alpha L w=K L_{w 1} / K L_{w 2}=13.156 / 11.840=1.111
$$

Average $(\alpha)$ for first in 5 yarns in double-layer fabrics (A):

$$
\alpha(A)==1.1993+1.111 / 2=1.155
$$

Second in $4-y$ arn $(B)$.

$K_{u p 1}=\operatorname{Cn} \sqrt{N}=0.04126(2 \times 10 \times 2.54) \sqrt{20.4}=9.4669 ; K_{u p 2}=0.04126 \times 18 \times 2.54 \times \sqrt{39.4}=11.84 ;$

$$
\alpha u p=K_{u p 1 /} K_{u p 2}=9.4669 / 11.84=0.7995
$$

$K L_{w 1}=0.04126(2 \times 10 \times 2.54) \sqrt{39.4}=13.156, K L_{w 2}=0.04126(18 \times 2.54) \sqrt{39.4}=11.840$

$$
\alpha L_{w}=K L_{w 1} / K L_{w 2}=13.156 / 11.840=1.111 .
$$

Average $(\alpha)$ for second area in 4 yarns $(B)$. In double-layer fabrics:

$$
\begin{gathered}
\alpha(B)=0.799+1.111 / 2=0.9552 \\
I F C=\frac{\sum(\propto \times W)}{N} \\
I F C=\sum(\alpha X W) / n=2(1.155+0.9552) / 2=1.055
\end{gathered}
$$

So, the length of yarn (warp-weft) in equation (4 A, B), first the length of warp extends between two intersection in perfect repeat of woven construction. Integration factor construction is an important material property which knowledge is required in various flow simulations while processing composites. This quantity is defined as the aesthetic, physical and mechanical properties, which characterizes the ease with which a fluid is forced to flow through the material under an applied pressure gradient. Both, the denting and stitches systems state and the impact integration factor construction measurement techniques were used in this work. The model higher standard was used as a process analysis tool. This enabled the user to determine such important process parameters as the location and type of injection ports, as well as the mechanical properties and location of the highphysical textile products. a process for a three-stiffener composite panel was proposed by using a denting system as shown in Table 2 (B, C, D). This array evolved from the variation of the process parameters while modeling several different composite panels. The modeling strategy, which was presented, provides a unified

Table 2: Specification of the denting system in reed [1]. approach towards an aesthetic, physical and mechanical properties and flow analysis of the textile reinforcement on a macro-scale of double layer woven fabrics. this approach allows a straightforward analysis of the reinforcement properties considering the variations in reinforcement denting and stitches systems, the aesthetic, physical properties, as well as also the material nonuniformity, as for example unbalanced crimp, deviations in the yarn cross sections area, and in spacing. An emphasis was made on the problem of resin flow through the reinforcement. a correlation relationship has been higher standard between the percentage of integration factor construction for double-layer fabrics and aesthetic, physical and mechanical properties, considering the use of the difference system of reed denting and stitches. We differentiated the system of reed denting by the 'integration factor construction' (IFC); the decrease in the (IFC) means that the open porosity for double-layer fabrics increases and the same time the air permeability also increases. If IFC $=2.488$ for a regular reed dents system with plain weave $1 / 1$ in a double-layer fabric then open porosity for the double-layer fabric is $21.025 \%$, and the air permeability is $38 \mathrm{Ft} 3 / \mathrm{cm}^{2}$. So, if we use the difference system of reed denting at fabric code B1, with plain weave $1 / 1$ for double-layer fabrics then we have: (A) 4 upper +2 lower: (B) 2 upper +1 lower with IFC $=2.0$, and the open porosity for this double-layer fabric will raise up to $26.15 \%$ with (42) air permeability $\mathrm{Ft} 3 / \mathrm{cm}^{2}$. This approach allows us taking into account the stacking and forming of the layers, both in the flow and structural analyses. In the case of flow analysis, this allows us accurately to study the effect of a multi-layer reinforcement structure of nesting deformation etc. on Area space. The lattice method should be chosen for a numerical analysis of flow through the reinforcement. In our case a specially designed interface, serving as a geometrical pre-processor, allows us reading the geometrical data the calculations were tested against problems of flow in open and filled channels, and flow through a parallel array of tows. The results have shown a very good agreement with theoretical and existing numerical data. This provides a basis for numerical

\begin{tabular}{|c|c|c|c|c|}
\hline \multirow{2}{*}{$\begin{array}{c}\text { Repeat } \\
\text { Wide /M.M }\end{array}$} & \multirow{2}{*}{\multicolumn{2}{|c|}{ Denting Systems }} & \multicolumn{2}{|r|}{ Distributing Yarns Systems } \\
\hline & & & Arias Code & Including Two Layers \\
\hline 2 & \multicolumn{2}{|c|}{ A } & A1 & 4:5: \\
\hline 2 & \multirow[t]{3}{*}{ B } & 1 & B1 & 6:3: \\
\hline 6 & & 2 & B2 & $6: 6: 6: 3: 3: 3:$ \\
\hline 12 & & 3 & B3 & $6: 6: 6: 6: 6: 6: 3: 3: 3: 3: 3: 3:$ \\
\hline 10 & \multirow[t]{3}{*}{ C } & 1 & $\mathrm{C} 1$ & $7: 6: 5: 4: 3: 2: 4: 5: 6:$ \\
\hline 12 & & 2 & $\mathrm{C} 2$ & 7: $7: 6: 6: 5: 5: 4: 4: 3: 3: 2: 2:$ and mirror copy \\
\hline 38 & & 3 & $\mathrm{C} 3$ & $7: 7: 7: 6: 6: 6: 5: 5: 5: 4: 4: 4: 3: 3: 3: 2: 2: 2:$ and mirror copy \\
\hline
\end{tabular}
analysis of Area space of more complex structures such as woven fabrics. The difference between the computed permeability for the cases of permeable and not permeable systems is within the range of $20 \ldots 30 \%$. The calculation enables achieving realistic quantitative results with a wide interval of uncertainty caused by variations of nesting of the layers (uncontrolled in the experimental procedure). 


\begin{tabular}{|c|c|c|c|c|}
\hline 24 & \multirow[t]{3}{*}{$\mathrm{D}$} & 1 & D1 & 7:7:0:7:6: 0:7:7:7:0:0:0: and mirror copy \\
\hline 31 & & \multirow{2}{*}{$\begin{array}{l}2 \\
3\end{array}$} & D2 & 7: 7: 7: 7: 7: 7: $0: 0: 7: 7: 7: 7: 0: 6: 6: 6: 6: 0: 6: 6: 6: 6: 6: 6:$ and mirror copy \\
\hline 26 & & & D3 & 7:7:7: 7: 7: 7:7:7:6:6: 0:0: and mirror copy \\
\hline
\end{tabular}

The measurements of mechanical properties of double woven fabrics are summarized in Table 2. The results were further statistically assessed using the analysis of variance to define the significance of the constructional parameters (type of weave and the method of stitching) on the selected mechanical properties. The results clearly indicate that wrinkle recovery is not altered by weave or the method of stitching. The method of stitching has a statistically important effect only on fabric stiffness in back warps direction and tearing strength in warp direction.

The importance of type of weave is much more evident regarding the breaking strength, breaking elongation, tearing strength and fabric stiffness. The effect of weave on breaking strength is important only in warp direction. This is probably the consequence of the yarn fineness used. In warp direction, the face and back yarns had different fineness's, while on the other hand the fineness of face and back weft was the same. So, when the yarn fineness of face and back fabric differs, the influence of weave becomes important. This well-known phenomenon arises from the fact that because of the crimp, the fabric strength is less than the strength of twisted yarns. If all other things are equal, plain weave fabrics which have the highest crimp will have the lowest strength. Our results show the following decreasing range of breaking strength with regard to the type of weave: twill-sateen-plain. Despite the fact that twill, sateen and hopsack weave all have the same number of thread passages in weave repeat of $6 \times 6$ threads, and so equal crimp, the breaking strength differs.

\section{Conclusion}

The objective of this study was to establish the dependencies between the integration factor construction of double layers woven fabrics, namely type of the method of stitching and denting, and aesthetic, physical and mechanical properties. The results of variance analysis clearly indicate that the denting and method of stitching doesn't have a statistically important effect at a $95 \%$ confidence level on the above-mentioned mechanical properties, except for the tearing strength in warp direction and fabric stiffness.
The influence of type of weave is much more evident regarding the mechanical properties. All the above-mentioned physical and mechanical properties are influenced by weave, except for wrinkle recovery. It is worth mentioning that all samples consisted of $100 \%$ cotton fabrics used for apparel and upholstery applications. The results of integration factor construction research could help the constructor in developing a new fabric construction with more appropriate properties [11].

\section{References}

1. Elnashar EA, Dubrovski P (2003) Macroporosity and air permeability in two-layer woven fabrics. World Textile Conference $-4^{\text {th }}$ AUTEX Conference, Roubaix, France.

2. Elnashar EA (2005) Volume porosity and permeability in double-layer woven fabrics. AUTEX Res J 5(4): 207-218.

3. Elnashar EA (1995) Effect of warp-ends densities distributions on some esthetical and physical properties of multi-layers woven fabric. MSc thesis, Faculty of applied arts, University of Helwan, Cairo, Egypt.

4. Elnashar EA (2006) The influence of woven and porosity on abrasion resistance of double-layers woven fabrics. International Workshop Physics of Fibrous Materials: Structure, Properties, Science Intensive Technologies and Materials.

5. Elnashar EA (2017) Smart multifunctional theory of clothes with using digital method. Int J Res Adv Eng Technol 3(2): 75-78.

6. Elnashar EA, Dubrovski PD (2008) The influence of the weave and the method of stitching on selected mechanical properties of woven double fabrics. AUTEX Res J 8(2): 41-43.

7. Elnashar EA (2012) Effect of fabric structure variation on garment aesthetic properties. Tekstil ve konfeksiyon 2: 132-136.

8. Jevšnik S, Rudolf A, Krašević S, Stjepanović Z, Saricam C, et al. (2010) Drapability-parameter for aesthetic appearance of garment. $2^{\text {nd }}$ Scientific conference: development trends in the textile industry design, technology, management. Pp. 76-80.

9. Jing ML (2006) Influence of structure parameters of lightweight worsted fabric on wearing characteristics. Wool Textile Journal 5: 48-51.

10. Szosland J (1999) Identification of structure of inter-thread channels in models of woven fabrics. Fibers and Textiles in Eastern Europe 7 (25): 41-45.

11. Zhang H, Zhu H, Zhang J (2003) Properties of fabric between bending, creasing and draping. Wool Textile Journal 3: 62-65. 